\title{
Pratiques
}

Linguistique, littérature, didactique

141-142 | 2009

La synonymie

\section{Présentation. Les paradoxes de la synonymie}

\section{Caroline Masseron}

\section{OpenEdition}

Journals

Édition électronique

URL : https://journals.openedition.org/pratiques/1260

DOI : $10.4000 /$ pratiques. 1260

ISSN : 2425-2042

\section{Éditeur}

Centre de recherche sur les médiations (CREM)

\section{Édition imprimée}

Date de publication : 15 juin 2009

Pagination : 3-8

\section{Référence électronique}

Caroline Masseron, «Présentation. Les paradoxes de la synonymie », Pratiques [En ligne], 141-142 I 2009, mis en ligne le 19 juin 2014, consulté le 12 mars 2023. URL : http://journals.openedition.org/ pratiques/1260 ; DOI : https://doi.org/10.4000/pratiques.1260 


\section{Les paradoxes de la synonymie}

SYNONYME : Se dit de deux ou plusieurs termes appartenant à la même catégorie (substantifs, adjectifs, verbes ou adverbes) et qui ont entre eux une analogie générale de sens, avec souvent des nuances différentes d'acception, particulières à chacun d'eux.

Cette définition extraite du Grand Larousse de la langue française (1978), est complétée par un article de spécialité duquel nous voudrions citer les lignes que voici :

Quelque conception que puissent adopter les grammairiens ou les linguistiques dont l'attention se porte sur les synonymes supra-segmentaux (paraphrases) ou infra-segmentaux (morphèmes), l'usage commun limite l'extension du terme aux mots de même sens, répertoriés dans des Dictionnaires des synonymes. Limitation pragmatique et non pas étymologique : les écrivains, et plus encore les scripteurs d'occasion, sont plus souvent à la recherche d'un mot que d'une phrase. Quand, plusieurs mots se présentant sous la plume, on se demande lequel convient, ou quand un mot, dont on sent intuitivement l'existence, se dérobe à la mémoire, on attend un secours du dictionnaire. Les synonymes sont du ressort de la lexicologie.

Une restriction supplémentaire, dans le cadre de la morphologie, est ordinairement apportée à la définition des synonymes : ils doivent être substituables l'un à l'autre, donc appartenir à la même classe grammaticale ; le synonyme d'un nom sera un nom, celui d'un adjectif, un adjectif, etc. Une nuance sémantique observée dans un adjectif (par exemple vieux dans mon vieil ami) ne se retrouve pas forcément dans le nom qui en dérive (la vieillesse); une nuance présente dans un verbe (courir les filles) peut se retrouver dans tel dérivé (coureur), non dans tel autre (course).

Les propriétés définitoires de la synonymie (domaine linguistique, nature de la relation, fonction, résidu problématique) ne sauraient être mieux énoncées. Reprenons-les : 
- Le domaine convoqué par la synonymie est celui de la sémantique lexicale: s'y rattache notamment l'attention portée à l'unité mot, étant donné l'association d'une forme et d'un sens que le mot symbolise (valeur d'usage) mieux que toute autre structure signifiante ;

- La reconnaissance d'une relation de sens (" une analogie générale de sens », le contenu « conceptuel » commun) entre deux ou plusieurs unités lexicales ;

- L'appartenance des synonymes à une même catégorie grammaticale, plus précisément, à une catégorie «lexicale» : noms, verbes, adjectifs et adverbes ;

- La fonction différentielle de la synonymie pour dissocier les acceptions d'une entrée polysémique, et pour s'interroger sur le statut du sens figuré (l'exemple de courir) ;

- Le test de la substituabilité entre les unités synonymes, et le rôle du cotexte syntagmatique pour rejeter ou accepter une commutation de termes;

- La discussion sur l'espace de recouvrement sémantique (synonymie absolue, partielle, ou approximative) entre les unités lexicales ; autrement dit, la possibilité de considérer des degrés de synonymie ou d'établir une échelle de valeurs ;

- L'analyse sémantique en composants noyaux (l'analogie générale, les traits fixant « la constante notionnelle ») et l'ajout de nuances ou d'acceptions particulières, lesquelles justifieraient l'existence de mots différents ainsi que l'impossibilité pour une langue de n'avoir aucun synonyme, ni de présenter des cas de synonymes absolus (c'est-à-dire interchangeables dans tous les contextes).

Enfin, la citation du GLLF rappelle à bon escient que si la synonymie pose quelques difficultés de délimitation théorique, d'un point de vue pragmatique, en revanche, elle est peu discutable, ainsi qu'en attestent les usages langagiers et le besoin, commun, de statuer sur le mot juste ${ }^{(1)}$. C'est ce paradoxe - la méfiance théorique mais un solide fondement empirique qui s'incarne dans les usages, les dictionnaires et la « réalité » du mot - qui nous a intrigués au premier chef, et incités à choisir la synonymie comme domaine problématique.

L'une des difficultés auxquelles se heurte la synonymie c'est si l'on peut dire la polyvalence de ses applications, voire leur disparité apparente. Parmi les plus remarquables d'entre elles et, il est vrai, très solidaires, rappelons l'histoire de la langue, la lexicographie et la traduction.

S'agissant de l'enseignement, par exemple, rappelons que la synonymie est une pratique - sinon une théorie - indispensable. Elle fait partie de ces ressources lexicales, spontanées ou travaillées, très importantes en production de texte, qu'il s'agisse d'opérer à un niveau paradigmatique (chercher un autre mot, plus adéquat à ce qu'on veut dire, plus précis, moins familier ou qui évitera une répétition, et remplacer le seul mot pour le moment disponible et actualisé), ou à un niveau syntagmatique et dans une visée qui demeure « stylistique » (l'effet d'insistance des binômes synonymiques); ou bien encore, qu'il s'agisse des interactions langagières dans la classe (maître - élèves) qui recourent à la synonymie (la feuille

(1) Rappelons à cet égard le titre de l'Abbé Girard: La justesse de la langue françoise ou les différentes significations des mots qui passent pour synonimes. 
ou la copie ; le rond ou le cercle), au même titre qu'à la paraphrase (visée explicative).

Au-delà des situations d'apprentissage, tout scripteur, quelles que soient sa maîtrise langagière et sa capacité métalinguistique à verbaliser un problème d'écriture lié au traitement synonymique, a rencontré la double question des synonymes : jugement d'équivalence sémantique ou recherche de l'unité manquante (chercher un synonyme pour dire..., qui aille avec..., pour remplacer...).

La synonymie fait donc symptomatiquement surgir deux lignes de fracture :

- Une problématique qui sera jugée plus ou moins pertinente, selon que l'analyse est indexée, dans le champ de la sémantique, à un paradigme ou à un autre, 1'un des clivages s'opérant, semble-t-il, par rapport à l'héritage saussurien (continuité ou rupture);

- Un modèle sémantique qui vise une description des effets de sens en réception, minorant du coup la prégnance des usages et des normes (Frei, Grammaire des fautes).

La présente livraison de Pratiques n'a d'autre but que de contribuer à éclaircir ce statut ambigu de la synonymie. L'économie générale du sommaire fait se succéder des contributions théoriques, qui empruntent leur conceptualisation à des modèles qui peuvent être concurrents, et des applications pratiques particulières sur des items lexicaux apparentés à des synonymes. D'une manière plus large, on conviendra à la lecture des articles que tous, en dépit des divergences théoriques (sémantique lexicale, sémantique cognitive ou constructivisme), font l'hypothèse que la synonymie peut constituer, au moins provisoirement, un analyseur intéressant des difficultés théoriques à décrire le sens des mots.

Dans son article, "La synonymie-“identité de sens" n'est pas un mythe ", Georges Kleiber, après avoir rappelé un autre paradoxe de la synonymie (« la relation la plus facile à définir, mais aussi la plus difficile à maintenir »), réfute les trois arguments (sémiotique, linguistique, et sémantique) qui sont fréquemment avancés pour discuter de l'existence de la synonymie. La réfutation de Kleiber souligne à plusieurs reprises que l'oubli du « mot» (du signe complet, les deux faces et les deux axes) a pu fausser les discussions sémantiques quand elles contestent la validité de la synonymie absolue comme relation d'identité de sens.

Pierre Cadiot pour sa part, dans un article intitulé « Couleur des mots ou synonymie », reprend, bien que sous un éclairage, on s'en doute, différent de celui de Kleiber, l'idée que la synonymie engage l'unité lexicale tout entière et sa valeur en discours. L'auteur note toutefois que la notion, inscrite dans une tradition de sémantique référentielle, perd de vue le motif du mot, lui préférant des acceptions limitées, c'est-à-dire réduisant la signification à un sens lexical isolé. Avec le concept de motif (Cadiot et Visetti 2001 : 97-127), c'est l'instabilité de la forme sémantique qui est soulignée, d'une part dans ses évolutions diachroniques et la possibilité d'une rétroaction sur un sens historique (train, habiller), et d'autre part dans sa composition hétérogène (la motivation née d'un composant morphologique, par exemple). Le motif désigne le noyau sémantique qui reste soumis à d'éventuels changements, dès lors que le mot est inséré en discours et profilé, stabilisé par le contexte. Ce cheminement conduit Cadiot à conclure, contrairement à Kleiber, qu'il n'y a pas de « synonymie au sens fort » et que la notion demeure peu compati- 
ble avec un modèle qui fonde une construction dynamique du sens sur la complexité et l'instabilité des motifs sémantiques.

Quant à Pierre Jalenques, il ébauche méthodiquement les étapes d'une analyse sémantique constructiviste en l'appliquant à deux verbes « synonymes ", laisser et garder. Les deux formes schématiques, distinctes, qui décrivent la construction du noyau sémantique de chaque verbe, conduisent l'auteur à réfuter la synonymie comme relation absolue et hors énoncé. S'il y a encore lieu de concevoir un lien synonymique, il faut lui réserver l'application locale, et partielle, de l'équivalence des deux énoncés (et non plus de deux unités lexicales).

Jacques François - Fléchage synonymique ou analyse componentielle dans l'examen de la polysémie verbale? Affecter vs compter - poursuit son investigation sémantaxique sur les verbes et la polysémie. Il confronte, au sujet de deux verbes polysémiques, affecter et compter, les résultats obtenus par des démarches et des outils différents. Les synonymes du dictionnaire électronique du CRISCO, par le biais d'un fléchage " continuiste », se montrent d'autant plus opératoires que le verbe présente une polysémie taxée d'hétérogène (affecter). L'outil syntaxique, qui discrimine des distributions et des traits syntaxiques, débouche sur un résultat contraire : il est mieux adapté au verbe à polysémie homogène (compter) ${ }^{(2)}$.

Ce numéro de Pratiques sur la synonymie intègre l'histoire de la langue et 1'analyse du discours lexicographique, grâce à un article de Gilles Petrequin - La synonymie au XVII ${ }^{\mathrm{e}}$ siècle : une évolution conceptuelle et pragmatique - qui interroge le dictionnaire de Richelet (1680). L'enquête à laquelle se livre l'auteur présente notamment l'intérêt de souligner le croisement qui s'opère en cette fin du $\mathrm{XVII}^{\mathrm{e}}$ siècle entre une conception rhétorique (les binômes synonymiques) et la genèse du discours lexicographique (le paradigme de synonymes en langue).

Denis Apothéloz, pour sa part, clôt provisoirement l'éventail des conceptions présentées, en se décentrant sur une question d'aspect, puisqu'il interroge « La quasi-synonymie du passé composé et du passé surcomposé existentiels ».

Enfin, le seul article qui s'appuie sur des discours effectifs, est celui de Michelle Lecolle - De la synonymie, vue à travers les emplois des mots synonyme, synonymie et synonymique dans les textes. L'auteur se fonde sur un corpus large pour établir ce que sont les représentations et usages communs de la notion, quand elle est envisagée par des non spécialistes. Le résultat est assez illustratif de ce qu'on pourrait identifier comme une figure de mots. En effet, nommer synonymes des mots qui n'en sont pas (par exemple profit et vol, sous la plume de Proudhon) revient au plan rhétorique à opérer un rapprochement paradoxal entre des notions plutôt antonymiques ou des réalités qu'apparemment tout sépare.

\section{Les traits discriminants (les nuances), ou le soutien des synonymes pour découvrir le noyau de sens original d'une unité lexicale}

Les contributions qui analysent des binômes particuliers ont en commun de tou-

(2) On trouvera en ligne, sur le site de Pratiques (http://www.pratiques-cresef.fr), une application de la méthode de Jacques François (2007), avec l'article de J.-C. Dunkhorst et C. Masseron: "Indice, signe et marque : exploration des liens synonymiques (DES) et configuration sémantique ». 
tes récuser la synonymie des unités retenues. D'une certaine façon, dans cette grande partie, on peut voir la synonymie comme une méthode opératoire, incluse dans un dispositif plus large et complexe de comparaison. Le synonyme, et sa non permutabilité, prend alors la fonction d'une sorte de catalyseur sémantique, pour sa vertu à faire apparaître un effet de sens de l'autre terme, mal perçu jusque-là. Les traits différenciateurs de K. Baldinger (1984) l'emportent.

C'est ainsi que, dans son article « Jalousie et envie, l'affectivité tout en nuances », Arkadiusz Koselak s'emploie à montrer que jalousie et envie représentent des affects contrastés, notamment pour l'axiologie, le rapport à l'objet et la relation des « actants ». Le recouvrement synonymique des deux termes envie et jalousie, étroit si l'on s'en tient au seul domaine affectif, offre un exemple de choix pour discuter du champ ouvert par le concept de volition, du besoin au désir. Les collocations de l'acception modale mériteraient, tout particulièrement, un examen approfondi (avoir envie/besoin de). Toujours au chapitre des applications, trois articles échappent à la catégorie nominale pour interroger la relation de synonymie. Dorota Sikora, "Arriver et venir - quand la deixis fait (et ne fait pas) la différence », s'appuie sur les données de la deixis pour dégager les propriétés sémantiques de chacun des deux verbes. Corinne Féron et Danielle Coltier, procèdent quant à elles à un examen de nature locutoire et vériconditionnelle (savoir et vérité) pour spécifier l' «Étude sémantique des unités censé / réputé / supposé 》 et contester la synonymie des unités choisies. De leur côté, Marie Lammert et Hélène Vassiliadou partent de l'adverbial type, dans l'ensemble, et examinent, différentiellement, les synonymes que livrent les dictionnaires. Le résultat des comparaisons entreprises engage des opérations sémantiques assez fondamentales : « de la généralité à la quantification totale en passant par l'approximation ». L'analyse de Sandrine Stein-Zintz revient à la catégorie nominale, même si l'on peut penser que certaines unités sont «plus nominales » que d'autres! Le trio examiné, part, partie et portion, laisse présager que la saisie de portion sera une saisie plénière pour un terme plutôt monosémique. La base commune de part et partie, au contraire, autorise l'hypothèse d'une saisie précoce et en quelque sorte, abstraite (l'opération de division, de partition), pour part et d'une polysémie supérieure pour chacun des termes.

L'entrée didactique du numéro ne présente qu'une seule contribution, celle de Claudine Garcia-Debanc et alii qui, postulant la nécessité d'apporter des aides lexicales à des élèves qui écrivent, développent l'exemple des verbes de déplacement dont la thématique est retenue pour une activité de classement (observation de la langue) et ensuite pour un travail de production écrite où les verbes étudiés sont réinvestis. À la relation de synonymie est préférée une typologie critériée des hyponymes de se déplacer. L'article témoigne, par les interrogations qu'il soulève, que le recensement et l'analyse des besoins didactiques (formation des mâ̂tres et compétence lexicale des élèves à renforcer) doivent être poursuivis.

Enfin, dans une notule bibliographique, Patrick Dendale clôt le dossier en répertoriant les articles de linguistique française qui ont été publiés dans la période récente et qui ont comme point commun de constituer des analyses contrastives (par exemple néanmoins, toutefois...).

Caroline Masseron 


\section{Indications bibliographiques}

BALDINGER, K. (1984) : Le problème de la synonymie : valeurs symboliques et valeurs symptomatiques, Vers une sémantique moderne, Chapitre 5, Paris, Klincksieck, 173-203.

CAdiot, P., \& Visetti, Y.-M. (2001) : Pour une théorie des formes sémantiques, Motifs, profils, thèmes, Paris, PUF.

FRANÇOIS, J. (2003) : La prédication verbale et les cadres prédicatifs, Louvain-Paris, Peeters.

- (2007) : Pour une cartographie de la polysémie verbale, Leuven-Paris, Peeters.

FREI, H. (1929, La grammaire des fautes, Genève, Slatkine Reprints.

GIRARD, ABBÉ G. ([1718, 1736, 1740] 1999) : La justesse de la langue françoise ou les différentes significations des mots qui passent pour synonimes, édition de M.-G. Adamo, Fasano-Paris, Schena-Didier Érudition.

GRAND LAROUSSE DE LA LANGUE FRANÇAISE (1978) : 7 Volumes, sous la dir. de L. Guilbert, R. Lagane et G. Niobey, Paris, Larousse.

Kleiber, G. (1999) : Problèmes de sémantique. La polysémie en question, Lille, Septentrion.

LAFAYE, B. ([1853] 1870) : Dictionnaire des synonymes de la langue française avec une introduction sur la théorie des synonymes, Paris, Librairie Hachette.

LE FRANÇAIS MODERNE (2007) : 1, La synonymie en questions. Échanges entre les époques, Françoise Berlan et Danielle Bouverot, dir., Paris, CILF.

MARTIN, R. (1976) : Inférence, antonymie et paraphrase, Paris, Klincksieck.

RouBAud, Abbé ([1785] 1796) : Synonymes français, 4 tomes, Paris, Bossange et alii.

Ullmann, S. ([1952] $\left.1965^{3}\right)$, Précis de sémantique française, Berne, Francke. 